\title{
Functional validation of novel compound heterozygous variants in B3GAT3 resulting in severe osteopenia and fractures: expanding the disease phenotype
}

Florian Job ${ }^{1 \dagger}$, Shuji Mizumoto ${ }^{2 \dagger}$, Laurie Smith ${ }^{3}$, Natario Couser ${ }^{3}$, Ashley Brazil $^{4}$, Howard Saal $^{4}$, Melanie Patterson $^{5}$, Margaret I. Gibson', Sarah Soden ${ }^{6}$, Neil Miller ${ }^{7}$, Isabelle Thiffault ${ }^{5}$, Carol Saunders ${ }^{5}$, Shuhei Yamada ${ }^{2}$, Katrin Hoffmann ${ }^{1}$, Kazuyuki Sugahara ${ }^{2,8^{*}}$ and Emily Farrow ${ }^{6,9^{*}}$ (D)

\begin{abstract}
Background: A new disease class of syndromes, described as linkeropathies, which are derived from defects in the glycosaminoglycan-linker region as well as glycosaminoglycan-side chains of proteoglycans is increasingly being recognized as a cause of human disease. Proteoglycans are an essential component of the extracellular matrix. Defects in the enzymatic process of proteoglycan synthesis broadly occur due to the incorrect addition of side chains. Previously, homozygous missense variants within the B3GAT3 gene encoding beta 1,3 glucuronyltransferase 3(GlCAT-I) responsible for the biosynthesis of glycosaminoglycans have been described in 7 individuals.

Case presentation: In this study, a 4-year-old patient with a severe phenotype of osteoporosis, hypotonia, joint laxity, fractures, scoliosis, biscuspid aortic valve and myopia was referred for next generation sequencing after extensive negative clinical testing. Whole exome sequencing was performed on the proband and his unaffected parents to identify the molecular basis of his disease. Sequencing revealed compound heterozygous variants in B3GAT3: c.1A > G (p.Met1?) and c.671 T>A (p.L224Q). Clinical and in vitro functional studies were then completed to verify the pathogenicity of the genotype and further characterize the functional basis of the patient's disease demonstrating the patient had a decrease both in the protein level of B3GAT3 and in the glucuronyltransferase activity when compared to control samples. Independent in vitro assessment of each variant confirmed the B3GAT3: c.1A > G (p.Met1?) variant is functionally null and the c.671 T>A (p.L224Q) missense variant has significantly reduced glucuronyltransferase activity ( $\sim 3 \%$ of control).

Conclusions: This is the first report of a patient with compound heterozygosity for a null variant in trans with a missense in B3GAT3 resulting in a severe phenotype, expanding both the genotypic and phenotypic spectrum of B3GAT3-related disease.
\end{abstract}

Keywords: B3GAT3, Glycosaminoglycan, Glucuronyltransferase-I, Linkeropathy, Proteoglycan

\footnotetext{
* Correspondence: k-sugar@sci.hokudai.ac.jp; egfarrow@cmh.edu

${ }^{\dagger}$ Equal contributors

${ }^{2}$ Department of Pathobiochemistry, Faculty of Pharmacy, Meijo University,

150 Yagotoyama, Tempaku-ku, Nagoya, Aichi 468-8503, Japan

${ }^{6}$ Department of Pediatrics, Children's Mercy Hospitals and Clinics, Kansas City,

MO, USA

Full list of author information is available at the end of the article
} 


\section{Background}

A new class of syndromes, described as linkeropathies, which encompass defects in the glycosaminoglycan (GAG)-linker region as well as GAG-side chains of proteoglycans is increasingly being recognized as the cause of human disease $[1,2]$. Proteoglycans are an essential component of extracellular matrix, particularly in cartilage, bone and the cardiovascular system [3-5]. They consist of a core protein produced in the endoplasmic reticulum, with a linker region that is added in the endoplasmic reticulum and Golgi apparatus [6]. The addition of disaccharide units, epimerisation and sulfation by various glycosyltransferases, sulfotransferases, and epimerases result in the biosynthesis of GAG side chains including heparan, chondroitin, and dermatan sulfates of proteoglycans $[1,7,8]$. Defects in any step of the enzymatic process of proteoglycan synthesis cause a decrease in mature GAGs [1, 2, 7]. Specifically, B3GAT3, beta1,3-glucuronyltransferase 3 (glucuronosyltransferase I, GlcAT-I), encodes a glycosyltransferase that functions to add the final glucuronic acid in the linker region tetrasaccharide, xylose-galactose-galactose-glucuronic acid, which is attached to specific serine residues on core proteins [9, 10]. Variants in B3GAT3 (Larsen Syndrome, OMIM \#245600), as well as B3GALT6 (Spondyloepimetapyseal Dysplasia with Join Laxity, OMIM \# 271640), B4GALT7 (Ehlers-Danlos syndrome with short stature and lim anomalies, OMIM \#130070), and XYLT1 (Desbuquois dysplasia-2, OMIM \#615777) have been associated with linkeropathies, which are broadly associated with joint laxity, fractures, and cardiac malformations [11-14]. However, these are a relatively new class of disorders, therefore it is unlikely the phenotypic spectrum has been fully recognized. Specifically, for B3GAT3, only 7 individuals, all with homozygous missense variants have been described ranging in phenotypic severity from mild to severe, with all patients having congenital heart defects, joint hypermobility, and fractures [11, 15-17].

Herein we describe the first patient with compound heterozygosity for a null variant in trans with a missense in B3GAT3 identified as the cause of disease and provide functional characterization.

\section{Materials and methods (See Additional file 1: Supplemental Information for Detailed Methods)} Whole exome sequencing

Samples were prepared for whole exome sequencing using the Nextera Rapid Capture Exome Kit (Illumina, San Diego, CA) according to manufacturer's protocols. Sequencing was completed on an Illumina HiSeq 2500 instrument with TruSeq v4 reagents, yielding paired end 125 nucleotide reads, with an average of $12.7 \mathrm{~GB}$ of data resulting in a mean $66 \times$ coverage. Gapped alignment to reference sequences (GRCh37.p5) was performed with
GSNAP and the GATK and analysis completed using custom-developed software, RUNES and VIKING as previously reported $[18,19]$.

\section{Cell culture}

Fibroblasts for cmh000720 utilized in the current studies were obtained when the patient was approximately 5 months old. Age- and sex-matched control fibroblasts were used for the functional assays.

\section{Western blotting}

Twenty micrograms of fibroblast cell lysate of patient and matched control were separated on a 10\% SDS-PA gel. Primary anti-B3GAT3 antibody (Abnova, Atlanta, GA) was for staining. As an additional loading control experiment, membranes were stripped and stained with anti-actin antibody (1:5000, polyclonal mouse; BD Bioscience).

\section{qPCR}

RNA was isolated from confluent primary fibroblast cultures using Trizol (Ambion/Thermo Fisher, Grand Island, NY). B3GAT3 mRNA levels were analyzed in primary fibroblasts by quantitative PCR (qPCR) using the ProtoScript ${ }^{\oplus}$ First Strand cDNA Synthesis Kit (NEB, Ipswich, MA). Two sets of intron spanning primers were designed for B3GAT3 mRNA (Additional file 1: Table S1) and GAPDH was used as an endogenous control. Relative expression was determined using the $2-\Delta \Delta \mathrm{Ct}$ method [20].

\section{GICAT-I assay of the recombinant wild-type and mutant, L224Q, GICAT-I proteins}

The expression vector of human GlcAT-I (wild-type), p3xFLAG-CMV8/hGlcAT-I, was used as described previously [11], and the mutant vector was constructed by overlapping extension PCR method [21]. Each vector was transiently transfected into HEK293T cells using the FuGENE HD DNA-transfection reagent (Promega, Madison, WI, USA). Three days after transfection, an aliquot of the conditioned media was individually incubated with an anti-FLAG affinity agarose resin (Wako, Osaka, Japan) at $4{ }^{\circ} \mathrm{C}$ for $4 \mathrm{~h}$. To examine the expression of both recombinant enzymes, SDS-PAGE and western blotting were performed using an anti-FLAG antibody.

Glucuronyltransferase activity was examined as described previously [11]. Briefly, the enzyme-bound resin as an enzyme source, UDP- $\left[{ }^{14} \mathrm{C}\right]-$ GlcA (Perkin Elmer, Boston, MA, USA) as the sugar donor substrate, and Galß1-3Galß1-O-methyl (Sigma, St. Louis, MO, USA) as the sugar acceptor were utilized for the assay. The reaction mixtures were incubated at $37{ }^{\circ} \mathrm{C}$ for $20 \mathrm{~min}$. The radiolabeled products were separated from UDP- $\left[{ }^{14} \mathrm{C}\right]$ GlcA using anion-exchange resin, AG 1-×8 ( $\mathrm{PO}_{4}^{2-}$ form), 
as described previously [22]. The isolated products, $\left[{ }^{14} \mathrm{C}\right]$-GlcA $\beta 1-3 \mathrm{Gal} \beta 1-3 \mathrm{Gal} \beta 1-O$-methyl, were quantified in a liquid scintillation counter (LSC-7400, HitachiAloka, Tokyo, Japan).

\section{Comparison of the GlcAT-I activities of fibroblast homogenates}

The homogenates of the fibroblasts were assayed using Galß1-3Galß1-O-methyl as an acceptor $(220 \mathrm{nmol})$ and UDP- $\left[{ }^{14} \mathrm{C}\right]-$ GlcA as a donor substrate, and then incubated for $4 \mathrm{~h}$ at $30{ }^{\circ} \mathrm{C}$. The procedures thereafter were described above.

\section{Cell-based ELISA}

Cell-based ELISA was carried out using the protocol provided by R\&D Systems Inc. (https://www.rndsystems.com/ products/cell-based-elisas) with slight modifications. Briefly, fibroblasts from the patient and a control subject were cultured to determine the amount of CS. Cells were washed and treated with chondroitinase $\mathrm{ABC}$ at $37^{\circ} \mathrm{C}$ for $30 \mathrm{~min}$. After washing the chondroitinase-treated cells were fixed, incubated with the primary antibodies, a mixture of commercial anti-CS-stub antibodies (1B5, 2B6, and 3B3) (Cosmo Bio Co., LTD, Tokyo, Japan). Subsequently the cells were incubated with a secondary antibody, alkaline phosphatase-conjugated anti-mouse IgG. Finally, the cells were incubated with the substrate, $p$-nitrophenyl phosphate, and analyzed by measuring the absorbance at $405 \mathrm{~nm}$ with an iMark microplate absorbance reader (Bio-Rad, Hercules, CA, USA).

\section{Characterization of the mutation in start codon of GIcAT-I} An expression vector of the C-terminally GFP-tagged human GlcAT-I, pAcGFP-hGlcAT-I (wild-type), was constructed. To examine the effects of variants in the start codon of GlcAT-I on the expression of the encoded proteins, mutant vectors were constructed in frame with C-terminal GFP tag. Each vector was transiently transfected into HEK293T as described above. To examine which ATG is utilized as the initiation codon, SDSPAGE and western blotting were performed using an anti-GFP antibody, mFX75 (Wako, Japan).

\section{Case presentation}

The patient, cmh000720, is currently a 6-year-old male who was referred for whole exome sequencing at 4 years of age. The patient was conceived via artificial insemination and was born at an estimated 38 weeks gestation via C-section to a 32-year-old G3P2 mother and distantly related 27-year-old father (5th cousins once removed). The pregnancy was complicated by gestational diabetes that was well managed with diet. Decreased fetal movements were noted throughout pregnancy, however the results from ultrasound exams and maternal serum AFP testing were normal. At birth the patient was noted to have a week cry, hypotonia, joint hypermobility, and an undescended testicle. Apgar scores were 8 and 9 at 1 and $5 \mathrm{~min}$ respectively; weight was $3.1 \mathrm{~kg}$ ( $30^{\text {th }} \%$ ile) ) and length $50.8 \mathrm{~cm}\left(68^{\text {th }} \%\right.$ ile). He was discharged home at 2 days of life. Newborn screening was reported to be normal. At a routine pediatric follow up appointment at two weeks of age the patient was hospitalized for additional evaluation due to concerns of his persistent low tone and cardiac murmur. At that time he was noted to have both a left hip dislocation and right hip subluxation, with bilateral acetabular dysplasia. Use of a Pavlik harness failed correct the hip dislocation due to profound ligamentous laxity. Echocardiogram revealed a PFO and biscupid aortic valve. The patient had inconsistent TSH levels and thus was treated with levothyroxine. An MRI was suggestive of an arachnoid cyst, but was otherwise unremarkable. At 2.5 months of age an EEG revealed abnormal sharp waves, and CSF glucose levels were found to be 38. Due to a concern for a GLUT1 deficiency, a ketogenic diet was trialed but discontinued after the patient became lethargic on the diet and repeat testing was normal. The patient has had no documented clinical seizure activity.

In addition to his hip dysplasia, cmh000720 was also noted to have significant kyphoscoliosis and osteopenia by 4 months of age. He has had multiple fractures associated with little to no trauma including his left tibia (12 months), right femur (19 months), right tibia (20 months), left femur (2.5 years), right femur shaft (3.75 years), distal right femur (3.8 years), and left femur (4.2 years). Pamidronate infusions were trialed but an increase in fractures was noted. He has significant osteoporosis; bone density by dexa of the left forearm at 4 years of age was measured as $\mathrm{Z}$ score of -5.8 . Spinal fusion rods were placed at 3 years of age and removed at 5 years of age. He is currently in a hard brace. Stabilization rods were also placed in both femurs (Fig. 1a).

The patient had developmental delay of gross motor milestones; currently he is able to walk with a walker and bear weight but cannot walk independently. Cognitively he is normal; he was able to say single words at 12 months and currently only has problems with articulation. At 6 years of age, in addition to the aforementioned symptoms, the patient is followed for mild aortic root and ascending aorta dilation, restrictive lung disease requiring C-PAP/BiPAP due to scoliosis and chronic obstructive pulmonary disease, bilateral hyperopia, astigmatism, amblyopia, and mild left ptosis, and hypoglycemia with illness. His weight $24.76 \mathrm{~kg}$ ( $85^{\text {th }_{2}} \%$ ile) and length $111 \mathrm{~cm}$ $\left(13^{\text {th }} \%\right.$ ile) are within normal limits, although he still receives supplemental nutrition overnight through a G-tube that was placed at 20 months of age. He is macrocephalic with an OFC of $54.8 \mathrm{~cm}\left(>98^{\text {th }} \%\right.$ ile). He is only mildly dysmorphic with downslanting palpebral fissures, bluish/grey 


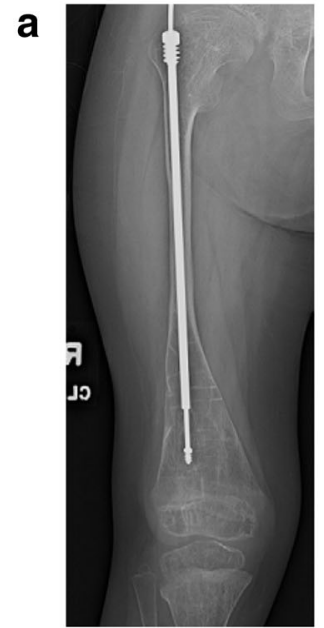

Rt. Femur

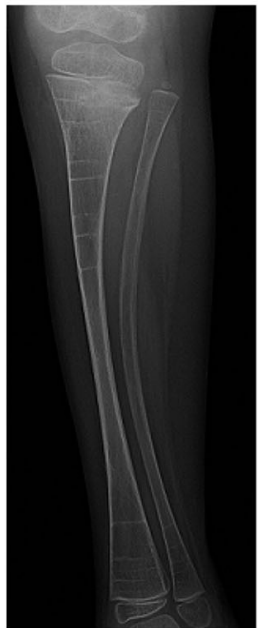

Rt. Tibia/Fibula

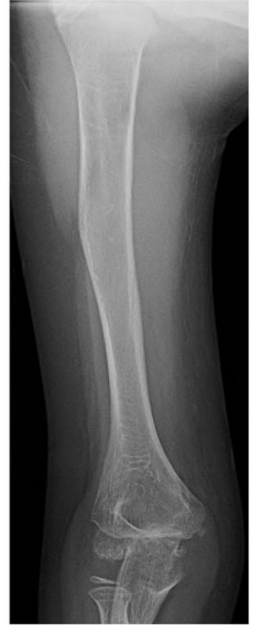

Rt. Humerus

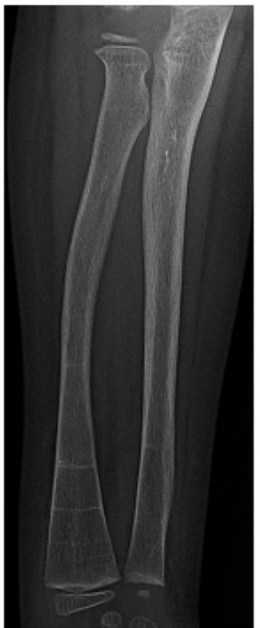

Rt. Radius/Ulna b

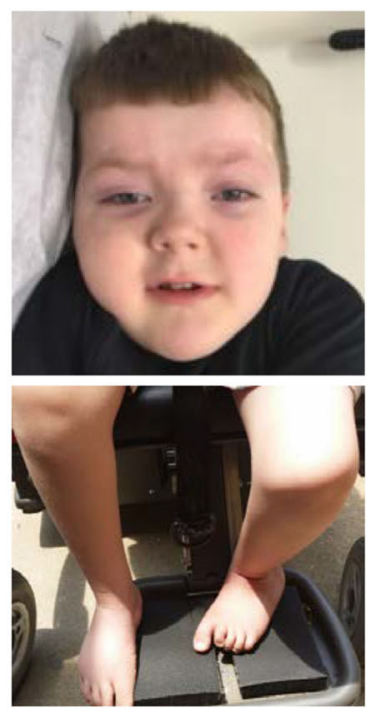

C

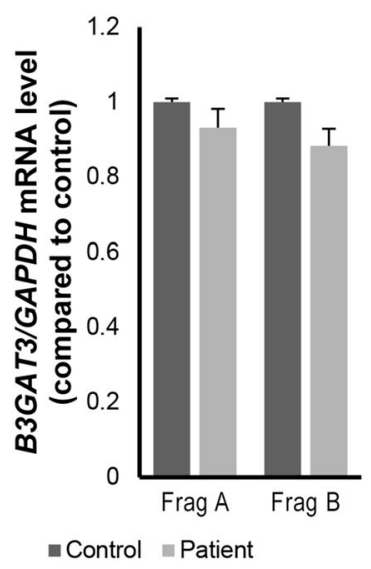

d
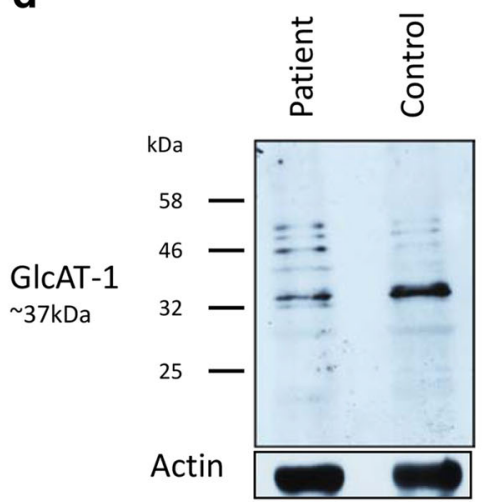

$\mathbf{e}$

Fig. 1 a X-rays revealed severely diffusely demineralized bones, along with multiple growth arrests lines. Stabilization rods are in both femurs and bilateral bowing of the tibiae and fibulae are evident (right pictured). b Cmh000720 is mildly dysmorphic with downslanting palpebral fissures (left), short neck, and curved lower extremities. While able to ambulate with a walker and physical assistance, he is wheelchair-bound. c mRNA expression of B3GAT3 in primary fibroblasts in cmh000720 was not statistically different from control using two sets of primers, whereas western blotting (d) showed a decrease in overall protein. e Glucuronyltransferase activity was determined by incorporation of $\left[{ }^{14} \mathrm{C}\right] \mathrm{Gl}$ CA into the Gal $\beta 1$ 3Galß1-O-methyl. The control sample utilized an enzyme source from the fibroblasts from a healthy control subject. ${ }^{*} P<0.0001$

sclera, a high arched palate, short neck, arachnodactyly, and hyperextensible skin with no striae (Fig. 1b). His bone age is estimated at 4 years 6 months ( $>2$ SD below expected for chronological age). He completed kindergarten with an IEP that includes occupational, speech and physical therapies.

The family history is notable for an older brother who was found to have a biscuspid aortic valve, after recommended screening. He has a healthy younger sister. Echocardiograms of both parents and younger sister were normal. Both parents are tall, and reportedly healthy.

\section{Results}

Prior to whole exome sequencing, the patient had extensive biochemical and genetic testing all of which were normal or non-diagnostic. Whole exome sequencing of the patient and both parents revealed compound heterozygous variants in B3GAT3: a paternally inherited c.1A > G (p.Met1?) and maternally inherited c.671 T $>$ A (p.L224Q). Subsequent Sanger sequencing of both siblings revealed that the older brother who also has a biscuspid aortic valve did not inherit either variant while the younger sister is heterozygous for the c.1A > G (p.M1?) variant (Additional file 1: Figure S1). While the c.1A > G variant has not been reported in any public database and is unique to the family in an internal database, a different variant at the same position has been reported in 2 heterozygous individuals in ExAC $(2 / 62,938)$. This variant is predicted to result in loss of translation initiation and was interpreted as likely pathogenic according to current ACMGG guidelines [23]. 
The maternally inherited c.671 T > A (p.L224Q) variant is in exon 4 and replaces a nonpolar leucine residue with a polar glutamine residue, a moderate physicochemical difference. The leucine is conserved across species (Additional file 1: Figure S2); and is predicted to be disease causing. The variant has not been reported in any public databases and is unique to the family in an internal database. Of note, a homozygous variant, G223S, has been reported in a child with a severe presentation of disease [16]. In the absence of further data, this variant was interpreted as a variant of unknown significance, however, as the patient's phenotype was consistent with a diagnosis of B3GAT3-related disease, functional studies were pursued to further characterize the patient's disease and novel variants.

To access the overall function of GlcAT-I, western blotting and expression analyses were performed using cultured primary fibroblasts from the patient and a healthy control. No difference in expression was detected in mRNA levels using two different primer pairs encompassing the first (Fragment A) and second (Fragment B) potential start codon (Fig. 1c, Additional file 1: Table S1). However western blotting demonstrated a significant decrease in protein levels (Fig. 1d). To examine glucuronyltransferase activity, assays of GlcAT-I activity were carried out using a cell lysate derived from the patient's fibroblast cells. In stark comparison to control cells, no GlcAT-I activity was detected when lysate from the patient's fibroblast cells was used as an enzyme source (Fig. 1e).

To further examine the effect of p.M1? and p.L224Q variants independently, wild-type and mutant vectors were constructed and glucuronyltransferase activity of the recombinant enzymes was tested. The GlcAT-IL224Q construct showed a marked reduction to $\sim 3 \%$ of the wild-type (Fig. 2a). However, protein levels were comparable to wild-type based on the western blotting (Fig. 2a). These results suggest that the L224Q variant affects the glucuronyltransferase activity and the biosynthesis of GAGs including chondroitin sulfate, dermatan sulfate, and heparan sulfate, and/or their proteoglycan forms.

To characterize the effects of the variant of the initiation codon on the encoded protein and to determine which alternative ATG may be utilized as the initiation codon in the open reading frame of the p.M1? gene, western blotting of C-terminally GFP-tagged mutant GlcAT-I proteins in all the possible reading frames (Additional file 1: Figure S4) was performed. Of note, there is not an in-frame alternative Met codon upstream of the Met1, therefore the c.1A > G variant is not predicted to result in an extended protein. Wild-type GlcAT-I was detected at a molecular weight of $\sim 64 \mathrm{kDa}$ (Fig. 2b). Although no obvious mutant proteins (No. $1 \sim$
3, 5, 7, and 8) of GlcAT-I were detected, GFP-tagged mutant proteins, \#4 as well as \#6, were clearly detected (Fig. 2b). These results indicate that the third and fifth ATGs of the open reading frame, at the positions of c.233 and c.584 in mutant B3GAT3 might be utilized, respectively, to initiate translation (Additional file 1: Figure S3 and Additional file 1: Figure S4, Additional file 1: Table S2). The resultant proteins bear a sequence totally unrelated to the GlcAT-I enzyme, thus the allele (not the proteins) is considered functionally null.

After confirming the p.M1? variant was functionally null, to analyze how the reduced activity of GlcAT-I from the p.L224Q variant affects the biosynthesis of chondroitin sulfate (CS) side chains of proteoglycans, the relative numbers of CS chains were analyzed by cellbased ELISA with three kinds of anti-CS-stub antibodies using fibroblast cells from the patient and control. These antibodies can be used as probes for investigating the relative numbers of $\mathrm{CS}$ chains on the core proteins of proteoglycans [11]. CS-stub antibodies showed a markedly reduced binding to the patient cells compared to control subject (Fig. 2c), suggesting that the number of CS chains was significantly reduced in the fibroblast cells from the patient.

\section{Discussion}

We have described the first patient of northern European descent with compound heterozygous variants in B3GAT3, expanding the phenotypic and genotypic spectrum associated with disease. Consistent with all patients reported to have B3GAT3-related disease, cmh000720 has a cardiac defects, joint laxity, dislocations, contractures, broad tips of fingers and a short neck [11, 15-17]. Our patient's features overlap with those of a patient with severe disease associated with a homozygous G223S variant; including multiple fractures, arachnodactyly, and blue sclerae. He did not have a diaphragmatic hernia or small thorax, but does have restrictive lung disease requiring $\mathrm{C}$-PAP/BiPAP at night. However, unlike other reported patients, our patient does not have short stature, currently his height is at the $13^{\text {th }}$ \%ile. Additionally, cmh000720 has macrocephaly, hypothryoidism and hypoglocemia, which have not been previously reported (Additional file 1: Table S3).

Independent in vitro studies demonstrated the p.M1? variant is functionally null and the p.L224Q variant has glucuronosyltransferase activity $\sim 3 \%$ of wild-type. Both of cmh000720's parents are healthy and no disease phenotype has been reported to date $[11,16,17]$. Consistent with the reported human phenotypes; null mice were embryonic lethal while heterozygous mice did not have a phenotype and were born at Mendelian frequencies [24]. Taken together, the data indicate that 50\% glucuronosyltransferase activity compared to wild-type is 
a

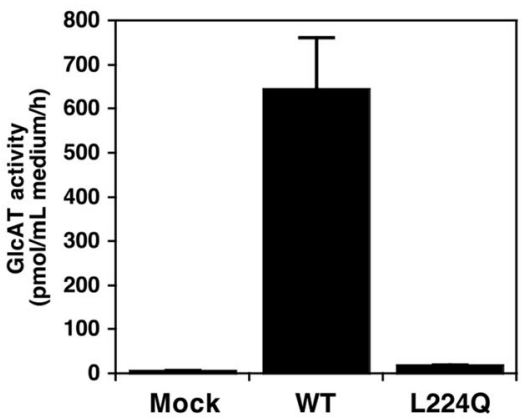

b

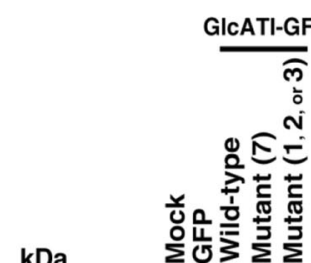

$k D a$

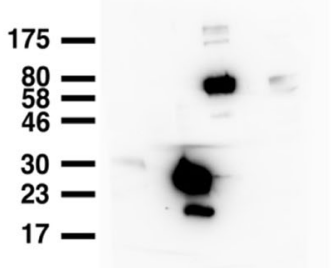

FLAG-

hGICAT-I

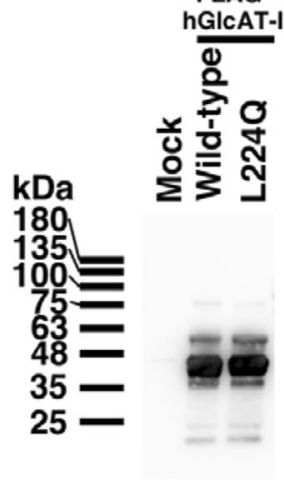

GICATI-GFP

$$
6
$$

0 于

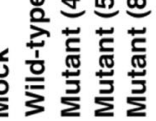

kDa

180

135

$100 \equiv$

$63=$

48-

35 -

$25-$

17 二

C

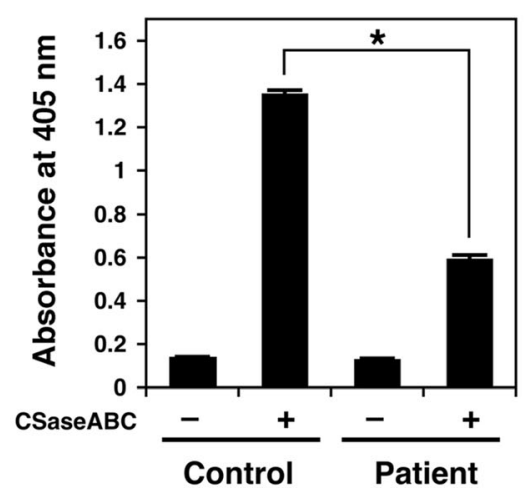

Fig. 2 a Glucuronyltransferase activity of each enzyme protein was examined using the partially purified recombinant enzymes, UDP- $\left[{ }^{14} \mathrm{C}\right]-\mathrm{Gl} / \mathrm{C}$ as the donor substrate, and Galß1-3Galß1-O-methyl as the acceptor substrate. Glucuronyltransferase activity was determined by incorporation of $\left[{ }^{14} \mathrm{C}\right] \mathrm{Gl}$ cA into the Galß1-3Galß1-O-methyl. "Mock" indicates the enzyme source from the HEK293T cells transfected with an empty vector. Values are the means \pm SE $(n=3)$. ${ }^{*}>0.0005$ versus wild-type was calculated by the ANOVA Dunnett test. Two sets of the mutant enzyme, L224Q, were prepared from two separate conditioned media from the HEK293T cell cultures (left). The purified recombinant GlcATI separated by SDS-PAGE was detected with the horseradish peroxidase-conjugated anti-FLAG antibody (Wako). The broad signals of the FLAG-tagged GlcAT-I may indicate the $\mathrm{N}$-glycosylated enzymes ( 40 kDa). Western blotting analysis showed the expression of comparable amounts of these recombinant proteins. (right). $\mathbf{b}$ The GFP-tagged GlCAT-I and its mutant proteins separated by SDS-PAGE were detected with anti-GFP antibody and the horseradish peroxidase-conjugated anti-mouse lgG antibody as the primary and secondary antibodies, respectively. The deduced molecular masses of GFP-tagged wild-type GlcAT-I and the mutant proteins (mutants \#1 8) were summarized in Additional file 1: Table S2. Although no obvious mutant proteins (No. $1 \sim 3,7$, and 8) of GlcAT-I were detected, GFP-tagged mutant proteins, \#4 as well as \#5 or 6, were clearly detected. Predicted molecular weights of the bands of \#5 and \#6 were 40.2 and $31.3 \mathrm{kDa}$, respectively (Additional file 1: Table S2). The GFP-tagged mutant protein \#5 or 6 was detected at a molecular weight of $\sim 30 \mathrm{kDa}$, suggesting that the observed GFP-tagged mutant protein might be mutant-\#6 rather than mutant-\#5. c Comparison of the amount of CS chains on the cell surface of fibroblasts from the patient and a healthy control by cell-based ELISA. $* P<0.0001$ 
sufficient for a normal phenotype. Further studies are needed to correlate the level of residual activity to resulting phenotype.

The cause of endocrine abnormalities in cmh000720 is not clear. Congenital hypothryoidism is relatively common, affecting $\sim 1 / 2000$ births. However, thyroid hormone has been shown to play a role in the expression of proteoglycans and so it cannot be ruled out that the patient's B3GAT3 disease is the underlying cause [25]. Similarly, hypoglycemia has not been reported in other patients. Previous studies have demonstrated a decrease in chondroitin sulfate and heparan sulfate in patients with diabetes [26], therefore it is possible that the overall decrease in GAGs is contributing to his hypoglycemia.

\section{Conclusion}

The linkeropathies are still a relatively new class of disorders, with the full disease spectrum yet to be defined. However, they should be considered as the differential diagnosis of patients with hypotonia, joint laxity, congenital heart disease and fractures. It is clear from the limited case reports to date that there is a wide spectrum of clinical severity associated with disease. As whole exome sequencing is quickly becoming the standard for clinical testing, these patients will likely be diagnosed earlier in their disease course. Further studies of additional patients are needed in order to fully elucidate the phenotypic spectrum and disease mechanisms of these disorders.

\section{Additional files}

Additional file 1: Supplemental Data- Detailed Methodology. Detailed description of methods presented in the manuscript. Figure S1. Sanger Sequencing. Description and traces of sanger confirmation of B3GAT3 variants. Figure S2. Computational Analysis of p.L224Q Variant. Computational modeling of wild-type and B3GAT3 p.L224Q variant, including amino acid conservation. Figure S3. Nucleotide and deduced amino acid sequences from the wild-type of the human GlcAT-I. Summary of potential alternative start codons within B3GAT3. Figure S4. Nucleotide and deduced amino acid sequences from the mutants of human GlCAT-I. Summary of putative variant proteins \#1-8 in B3GAT3. Table S1. qPCR primers for GIATC-I mRNA Expression in Primary fibroblasts. Table of qPCR primer sequences. Table S2. The deduced molecular masses of the GFP-tagged wild-type GlCAT-I and the mutant proteins (mutants \#1 8). Summary of predicted molecular weight of mutant proteins. Table S3. Primers for construction of the GFP-tagged hGlcAT-I expression vectors. PCR Primers for construction of vectors. Table S4. Phenotype Comparison of reported patients with B3GAT3 related disease. Phenotypic comparison of published patients with B3GAT3-related disease. (DOCX $736 \mathrm{~kb}$ )

\section{Acknowledgements}

We would like to thank the family for their participation and support of this publication. We would also like to thank Suzanne Herd, study coordinator, for her assistance.

\section{Funding}

This work was supported in part by the Marion Merrell Dow Foundation, Children's Mercy Hospital Kansas City, and the William T. Kemper Foundation, a Grant-in-Aid for Scientific Research (C) 16 K08251 (to S. M.) from the Japan Society for the Promotion of Science, Japan; by Grant-in-Aid for Challenging Scientific Research from the Research Institute of Meijo University (to S. M.); by the Nakatomi Foundation (to S. M.); by Practical Research Project for Rare/ Intractable Diseases \#105 (to S. Y.) from Japan Agency for Medical Research and Development (AMED)

\section{Availability of data and materials}

All data for this study is presented in the article and Additional file 1.

\section{Authors' contributions}

The author(s) have made the following declarations about their contributions: Conceived and designed the experiments: FJ, SM, IT, CS, KH, KS, EG. Contributed reagents/materials/analysis tools: NM, EF, SY, KH, KS. Wrote the paper: EF, FJ, SM, KS. Reviewed the manuscript: FJ, SM, LS, NC, AB, $H S, M P, M G, S S, N M, I T, C S, S Y, K H, K S$, EF. Contributed to the recruitment and clinical investigations of the patient for the study: SS, EF, AB, HS, LS, NC. Performed the functional analysis: FJ, SM. All authors read and approved the final manuscript.

\section{Competing interests}

The authors declare that they have no competing interests.

\section{Consent for publication}

Informed assent/consent was obtained for all family members for case presentation and publication.

\section{Ethics approval and consent to participate}

All studies were approved by the Institutional Review Board at Children's Mercy Hospitals and Clinics, Kansas City, Missouri (IRB \#11120514) as well as the local ethics committee from Meijo University, Nagoya, Japan. Informed assent/consent was obtained for all family members for participation in the described research studies. Both parents consented for the participation of all children.

\section{Author details}

${ }^{1}$ Institute for Human Genetics and Molecular Biology, Martin Luther University Halle-Wittenberg, Magdeburger Str. 2, 06112 Halle (Saale), Germany. ${ }^{2}$ Department of Pathobiochemistry, Faculty of Pharmacy, Meijo University, 150 Yagotoyama, Tempaku-ku, Nagoya, Aichi 468-8503, Japan. ${ }^{3}$ University of North Carolina School of Medicine, Division of Pediatric Genetics and Metabolism, Department of Pediatrics, Raleigh, NC, USA. ${ }^{4}$ Division of Human Genetics, Cincinnati Children's Hospital Medical Center, Cincinnati, OH 45229, USA. ${ }^{5}$ Department of Pathology, Children's Mercy Hospitals and Clinics, Kansas City, MO, USA. ${ }^{6}$ Department of Pediatrics, Children's Mercy Hospitals and Clinics, Kansas City, MO, USA. ${ }^{7}$ Department of Medical Informatics, Children's Mercy Hospitals and Clinics, Kansas City, MO, USA. ${ }^{8}$ The Laboratory of Proteoglycan Signaling and Therapeutics, Graduate School of Life Science, Faculty of Advanced Life Science, Hokkaido University, Sapporo 001-0021, Japan. ${ }^{9}$ Center for Pediatric Genomic Medicine, Children's Mercy Hospitals and Clinics, 2420 Pershing, Suite 100, Kansas City, MO, USA.

Received: 22 June 2016 Accepted: 6 November 2016

Published online: 21 November 2016

\section{References}

1. Mizumoto S, Ikegawa S, Sugahara K. Human genetic disorders caused by mutations in genes encoding biosynthetic enzymes for sulfated glycosaminoglycans. J Biol Chem. 2013;288(16):10953-61.

2. Mizumoto S, Yamada S, Sugahara K. Mutations in biosynthetic enzymes for the protein linker region of chondroitin/dermatan/heparan sulfate cause skeletal and skin dysplasias. Biomed Res Int. 2015:2015:861752.

3. Bulik DA, Wei G, Toyoda H, Kinoshita-Toyoda A, Waldrip WR, Esko JD, Robbins PW, Selleck SB. sqv-3, -7 , and -8, a set of genes affecting morphogenesis in Caenorhabditis elegans, encode enzymes required for glycosaminoglycan biosynthesis. Proc Natl Acad Sci U S A. 2000;97(20):10838-43.

4. Iozzo RV. Matrix proteoglycans: from molecular design to cellular function. Annu Rev Biochem. 1998;67:609-52.

5. Sugahara K, Mikami T, Uyama T, Mizuguchi S, Nomura K, Kitagawa H. Recent advances in the structural biology of chondroitin sulfate and dermatan sulfate. Curr Opin Struct Biol. 2003;13(5):612-20.

6. Dick G, Akslen-Hoel LK, Grondahl F, Kjos I, Prydz K. Proteoglycan synthesis and Golgi organization in polarized epithelial cells. J Histochem Cytochem. 2012;60(12):926-35. 
7. Bishop JR, Schuksz M, Esko JD. Heparan sulphate proteoglycans fine-tune mammalian physiology. Nature. 2007:446(7139):1030-7.

8. Mikami T, Kitagawa H. Biosynthesis and function of chondroitin sulfate. Biochim Biophys Acta. 2013;1830(10):4719-33.

9. Kitagawa H, Tone Y, Tamura J, Neumann KW, Ogawa T, Oka S, Kawasaki T, Sugahara K. Molecular cloning and expression of glucuronyltransferase I involved in the biosynthesis of the glycosaminoglycan-protein linkage region of proteoglycans. J Biol Chem. 1998;273(12):6615-8.

10. Pedersen LC, Tsuchida K, Kitagawa H, Sugahara K, Darden TA, Negishi M. Heparan/chondroitin sulfate biosynthesis. Structure and mechanism of human glucuronyltransferase I. J Biol Chem. 2000;275(44):34580-5.

11. Baasanjav S, Al-Gazali L, Hashiguchi T, Mizumoto S, Fischer B, Horn D, Seelow $D$, Ali BR, Aziz SA, Langer R, et al. Faulty initiation of proteoglycan synthesis causes cardiac and joint defects. Am J Hum Genet. 2011;89(1):15-27.

12. Faiyaz-Ul-Haque M, Zaidi SH, Al-Ali M, Al-Mureikhi MS, Kennedy S, Al-Thani G, Tsui LC, Teebi AS. A novel missense mutation in the galactosyltransferase-I (B4GALT7) gene in a family exhibiting facioskeletal anomalies and Ehlers-Danlos syndrome resembling the progeroid type. Am J Med Genet A. 2004;128A(1):39-45

13. Nakajima M, Mizumoto $\mathrm{S}$, Miyake $\mathrm{N}$, Kogawa R, lida A, Ito H, Kitoh H, Hirayama A, Mitsubuchi H, Miyazaki O, et al. Mutations in B3GALT6, which encodes a glycosaminoglycan linker region enzyme, cause a spectrum of skeletal and connective tissue disorders. Am J Hum Genet. 2013;92(6):927-34.

14. Schreml J, Durmaz B, Cogulu O, Keupp K, Beleggia F, Pohl E, Milz E, Coker M, Ucar SK, Nurnberg G, et al. The missing "link": an autosomal recessive short stature syndrome caused by a hypofunctional XYLT1 mutation. Hum Genet. 2014;133(1):29-39.

15. Budde BS, Mizumoto S, Kogawa R, Becker C, Altmuller J, Thiele H, Ruschendorf F, Toliat MR, Kaleschke G, Hammerle JM, et al. Skeletal dysplasia in a consanguineous clan from the island of Nias/Indonesia is caused by a novel mutation in B3GAT3. Hum Genet. 2015;134(7):691-704.

16. Jones KL, Schwarze U, Adam MP, Byers PH, Mefford HC. A homozygous B3GAT3 mutation causes a severe syndrome with multiple fractures, expanding the phenotype of linkeropathy syndromes. Am J Med Genet A. 2015;167A(11):2691-6.

17. von Oettingen JE, Tan WH, Dauber A. Skeletal dysplasia, global developmental delay, and multiple congenital anomalies in a 5-year-old boy-report of the second family with B3GAT3 mutation and expansion of the phenotype. Am J Med Genet A. 2014;164A(6):1580-6.

18. Saunders CJ, Miller NA, Soden SE, Dinwiddie DL, Noll A, Alnadi NA, Andraws $\mathrm{N}$, Patterson ML, Krivohlavek LA, Fellis J, et al. Rapid whole-genome sequencing for genetic disease diagnosis in neonatal intensive care units. Sci Transl Med. 2012;4(154):154ra135.

19. Soden SE, Saunders CJ, Willig LK, Farrow EG, Smith LD, Petrikin JE, LePichon JB, Miller NA, Thiffault I, Dinwiddie DL, et al. Effectiveness of exome and genome sequencing guided by acuity of illness for diagnosis of neurodevelopmental disorders. Sci Transl Med. 2014;6(265):265ra168.

20. Livak KJ, Schmittgen TD. Analysis of relative gene expression data using real-time quantitative PCR and the 2(-Delta Delta C(T)) Method. Methods. 2001;25(4):402-8.

21. Zhao S, Yamamoto R. Detection of Mycoplasma meleagridis by polymerase chain reaction. Vet Microbiol. 1993;36(1-2):91-7.

22. Tone Y, Kitagawa H, Imiya K, Oka S, Kawasaki T, Sugahara K. Characterization of recombinant human glucuronyltransferase I involved in the biosynthesis of the glycosaminoglycan-protein linkage region of proteoglycans. FEBS Lett. 1999;459(3):415-20.

23. Richards S, Aziz N, Bale S, Bick D, Das S, Gastier-Foster J, Grody WW, Hegde $M$, Lyon E, Spector E, et al. Standards and guidelines for the interpretation of sequence variants: a joint consensus recommendation of the American College of Medical Genetics and Genomics and the Association for Molecular Pathology. Genet Med. 2015;17(5):405-24.

24. Izumikawa T, Kanagawa N, Watamoto Y, Okada M, Saeki M, Sakano M, Sugahara K, Sugihara K, Asano M, Kitagawa H. Impairment of embryonic cell division and glycosaminoglycan biosynthesis in glucuronyltransferase--deficient mice. J Biol Chem. 2010;285(16):12190-6.

25. Bassett JH, Swinhoe R, Chassande O, Samarut J, Williams GR. Thyroid hormone regulates heparan sulfate proteoglycan expression in the growth plate. Endocrinology. 2006;147(1):295-305.

26. Gowd V, Gurukar A, Chilkunda ND. Glycosaminoglycan remodeling during diabetes and the role of dietary factors in their modulation. World J Diabetes. 2016;7(4):67-73.

\section{Submit your next manuscript to BioMed Central and we will help you at every step:}

- We accept pre-submission inquiries

- Our selector tool helps you to find the most relevant journal

- We provide round the clock customer support

- Convenient online submission

- Thorough peer review

- Inclusion in PubMed and all major indexing services

- Maximum visibility for your research

Submit your manuscript at www.biomedcentral.com/submit
Biomed Central 